\title{
Sarcoma indiferenciado primário no sistema nervoso central
}

Primeira submissão em 10/10/11 Última submissão em 10/10/11 Aceito para publicação em 06/12/11 Publicado em 20/04/12

\section{Primary undifferentiated sarcoma of the central nervous system}

Milton Marcio Machota Juniori; Arthur Cornelian Gentili²; Fabricio Machado Marques ${ }^{3}$; Paulo Roberto Maciel ${ }^{4}$; Silvio Atsushi Ogata ${ }^{5}$; Luiz Fernando Bleggi-Torres ${ }^{6}$

\begin{tabular}{l|l}
\multicolumn{1}{c|}{ unitermos } & resUmo \\
Primário & $\begin{array}{l}\text { Introdução: O sarcoma de sistema nervoso central (SNC) é uma neoplasia rara, com incidência de } \\
\text { Sarcoma }\end{array}$ \\
Sistema nervoso central \\
maioria é tratada com ressecção radical. Relato: Homem, 29 anos, com episódios de crises convulsivas \\
e diagnóstico de hemorragia intraparenquimatosa. Durante a cirurgia, foi identificada lesão bem \\
delimitada. A histologia demonstrou neoplasia fusocelular com atipias e numerosas mitoses. Os únicos \\
marcadores imuno-histoquímicos positivos foram vimentina e S-100. O diagnóstico foi de sarcoma \\
indiferenciado de alto grau. Conclusão: No diagnóstico diferencial de sarcomas de SNC, devem-se \\
excluir lesões metastáticas e gliossarcoma.
\end{tabular}

abstract

Introduction: The central nervous system (CNS) sarcoma is a rare neoplasm with an incidence of $0.1 \%$ to $4.3 \%$ in intracranial tumors. They are aggressive with poor prognosis, and mostly treated with radical resection. Report: 29 year-old male patient with episodes of seizures and diagnosed with intraparenchymal hemorrhage. During the surgery a well-defined lesion was identified. Histology showed a spindle cell neoplasm with atypia and numerous mitoses. The immunohistochemical markers were positive only for vimentin and S-100. The diagnosis was high-grade undifferentiated sarcoma. Conclusion: Metastatic lesions and gliosarcoma should be excluded in the differential diagnosis of CNS sarcomas. key words

Primary

Sarcoma

Central nervous system

\footnotetext{
1. Médico-residente em Anatomia Patológica do Hospital das Clínicas da Universidade Federal do Paraná (HC-UFPR).

2. Médico-residente em Anatomia Patológica do HC-UFPR.

3. Médico-residente em Anatomia Patológica do HC-UFPR.

4. Médico-residente em Radiologia do HC-UFPR.

5. Especialista em Radiologia; radiologista do HC-UFPR.

6. Doutor em Neuropatologia; professor titular do Departamento de Patologia Médica da UFPR.
} 


\section{Introdução}

O sarcoma de sistema nervoso central (SNC) é uma neoplasia rara, com incidência de $0,1 \%$ a $4,3 \%$ dos tumores intracranianos ${ }^{(6)}$. São tumores agressivos com prognóstico reservado que podem surgir do parênquima cerebral ou das meninges $^{(1)}$. Não há consenso sobre o melhor tratamento para essas lesões. Alguns trabalhos advogam ressecção radical como tratamento de escolha, não se sabendo ao certo a utilidade de terapias adjuvantes, como radioterapia e quimioterapia ${ }^{(6)}$.

No presente relato, apresentamos o caso de um paciente de 29 anos com história de hemorragia intraparenquimatosa cerebral. Durante a abordagem cirúrgica e a posterior avaliação histológica, constatou-se tratar de um sarcoma de SNC.

\section{Caso}

No dia 7 de abril de 2011, um homem de 29 anos apresentou quadro de síncope e convulsão tônico-clônica generalizada. Na avaliação inicial, apresentava-se em período pós-ictal com sonolência, confusão mental, parestesia em hemiface esquerda, vertigem, disartria, turvamento visual e ataxia. A tomografia computadorizada (TC) de crânio evidenciou hemorragia intraparenquimatosa em região occipital direita (Figura 1). O paciente tinha histórico de linfoma tratado com quimioterapia há 12 anos; não tinha antecedentes de tabagismo, hipertensão ou diabetes. Hemograma, tempo de atividade de protrombina (TAP) e tempo de tromboplastina parcial ativado estavam dentro da normalidade. A ressonância magnética (RM) revelava hemorragia intraparenquimatosa parieto-occipital direita com $3 \times 2,9 \times 2 \mathrm{~cm}$ e suspeita de malformação vascular. $\mathrm{Na}$ investigação com arteriografia, não foram visualizadas fístulas ou malformações vasculares. Optou-se, então, pelo tratamento conservador com carbamazepina e o paciente foi encaminhado para acompanhamento ambulatorial.

No dia 21 de abril de 2011, o paciente retornou com cefaleia intensa e novo episódio convulsivo. Foi realizada nova TC, a qual evidenciou aumento do hematoma e sinais de ressangramento associado a efeito de massa e desvio de linha média. Decidiu-se, então, realizar abordagem cirúrgica para drenagem do hematoma. No dia 3 de maio, durante o procedimento cirúrgico foi constatada, após drenagem do hematoma, lesão arredondada, firme, acinzentada, vascularizada e com plano de clivagem, que foi ressecada.

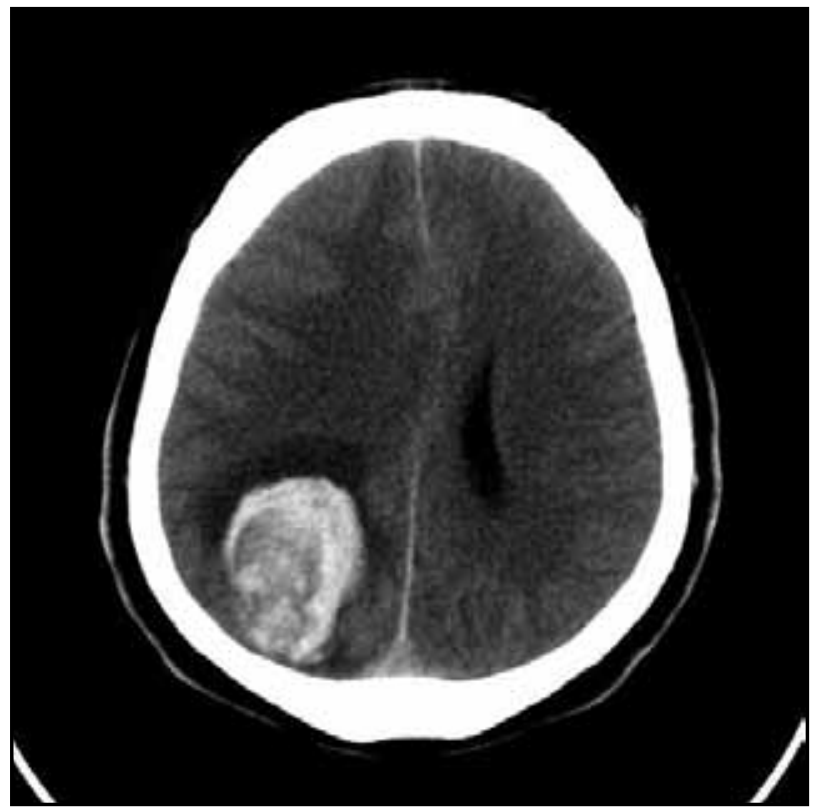

Figura 1 - Tomografia de crânio mostrando lesão intra-axial hiperdensa, bem delimitada em região occipital direita, associada ao edema do tecido nervoso adjacente, consistente com hemorragia intraparenquimatosa

O exame anatomopatológico peroperatório sugeriu a possibilidade de melanoma. No pós-operatório, o paciente foi submetido a avaliação dermatológica e exame de fundo de olho, que não constataram lesões sugestivas de melanoma. As TCs de tórax, abdômen e pelve não revelaram outras lesões. No dia 9 de maio, o paciente recebeu alta e estava em acompanhamento para realização de radioterapia.

$\mathrm{Na}$ avaliação histológica, a neoplasia era hipercelular, fasciculada, com células fusiformes atípicas com numerosas mitoses (38 por 10 campos de grande aumento) e necrose ausente. Na coloração para reticulina, notou-se formação de padrão reticular pericelular. No exame imuno-histoquímico, a neoplasia era positiva para vimentina e S-100. Os marcadores antígeno antiepitelial da membrana (antiEMA), proteína glial fibrilar ácida (GFAP), human melanoma black-45 (HMB-45), melan-A, desmina, CD34, fator VIII, $\mathrm{BCl}-2$ e CD99 foram todos negativos. O índice proliferativo pelo Ki-67 foi de 95\% (Figuras 2 a 5). Tendo em vista o quadro morfológico e imuno-histoquímico, o diagnóstico foi de sarcoma indiferenciado de alto grau.

\section{Discussão}

O sarcoma primário de SNC possui incidência que varia entre $0,1 \%$ e $4,3 \%$ de todas as neoplasias do cérebro. É mais frequente em crianças e jovens, apesar de poder ocorrer em qualquer faixa etária ${ }^{(4-6,8)}$. 


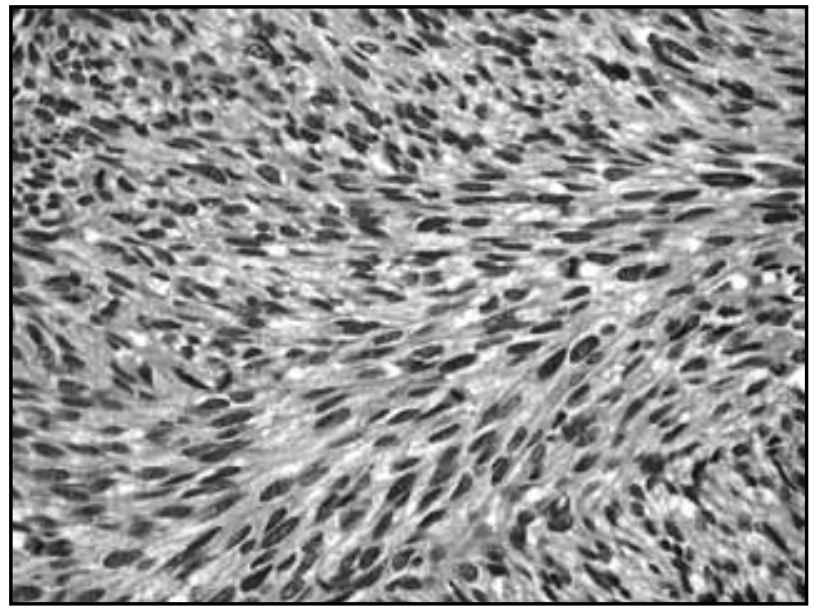

Figura 2 - Microscopia: padrão de crescimento fusocelular com feixes curtos e alta taxa mitótica (HE, 400x)

$H E$ : hematoxilina e eosina.

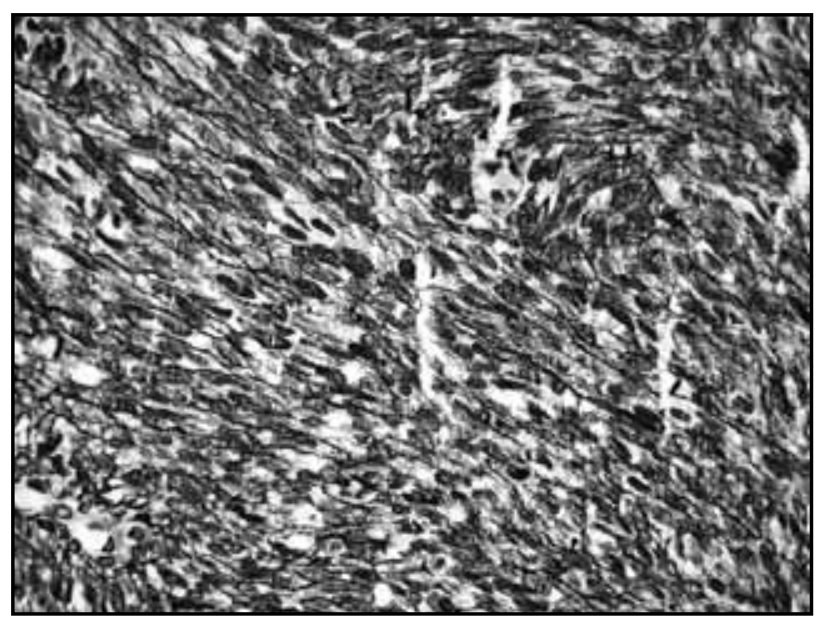

Figura 3 - Deposição de reticulina pericelular (Reticulina, 400x)

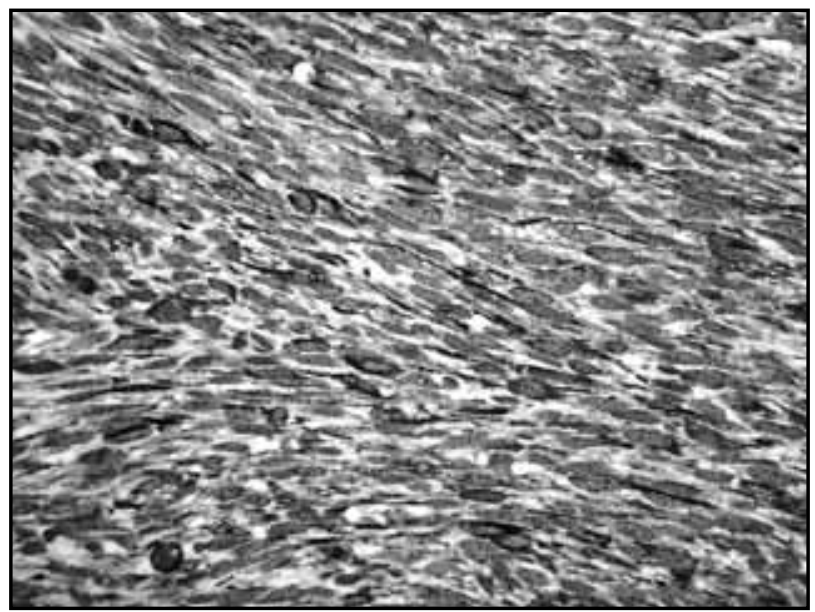

Figura 4 - Imuno-histoquímica antivimentina, com marcação forte e difusa (vimentina, 400x)

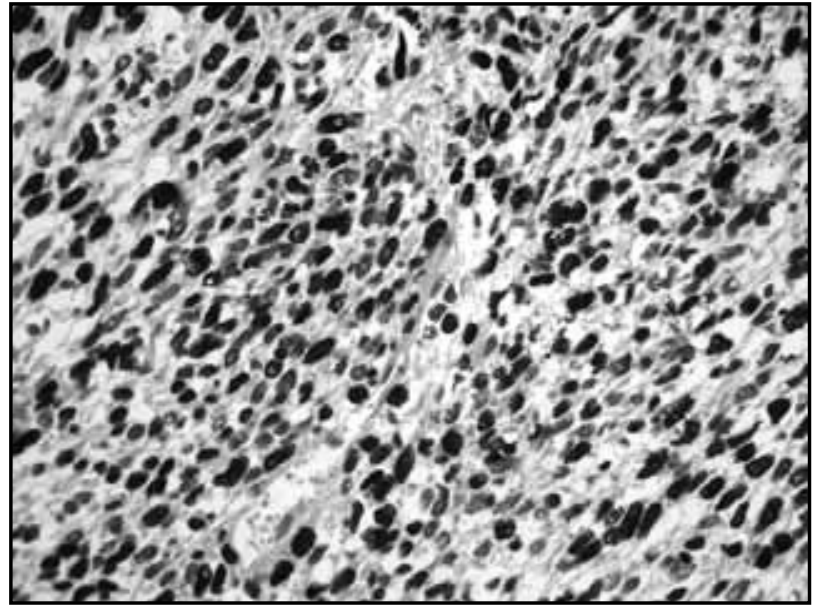

Figura 5 - Alto índice proliferativo com antiKi-67 (Ki-67, 400x)

A etiologia desse tumor é controversa e vários fatores estão implicados, como fatores genéticos, trauma (incluindo intervenções cirúrgicas prévias), vírus, agentes químicos e radiação. Em nosso relato, o paciente possuía histórico de quimioterapia para tratamento de linfoma 12 anos antes do desenvolvimento da lesão. Mubarak et al. ${ }^{(6)}$ relataram o caso de uma criança submetida a quimioterapia e radioterapia com desenvolvimento de sarcoma de SNC após 10 anos de evolução. Postula-se que essa neoplasia se origina em células mesenquimais imaturas pluripotenciais presentes na dura-máter, nas leptomeninges, nos espaços periadventícios e no estroma do plexo coroide ${ }^{(2-6,8)}$

Na avaliação radiológica, não há achados característicos que auxiliem na distinção dos sarcomas de outras neoplasias, afecções inflamatórias ou infecciosas do SNC $(3,6)$. McDonald et al.(4) relataram o caso de um homem de 43 anos que apresentou quadro de hemorragia intra-axial em região parieto-occipital direita, constatando-se fibrossarcoma primário de SNC. Em nosso caso, o paciente apresentou quadro de hemorragia intraparenquimatosa e somente depois da abordagem cirúrgica e do exame histológico foi diagnosticado sarcoma.

O sarcoma primário de SNC é raro e sua tipificação histológica nem sempre é clara(4). Em uma série de 66 sarcomas de SNC da Mayo Clinic, 27\% dos tumores eram classificados como sarcomas indiferenciados, seguidos pelos diagnósticos de fibrossarcoma (26\%), fibro-histiocitoma maligno $(21 \%)$ e outros subtipos $(26 \%)^{(8)}$. Neste relato, o diagnóstico foi de sarcoma indiferenciado, com base no padrão fusocelular com feixes curtos, fasciculado, no aspecto do arcabouço reticulínico e na positividade para vimentina à imuno-histoquímica. Além disso, a negatividade para EMA e GFAP afasta os diagnósticos de sarcoma meníngeo 
e gliossarcoma. Os achados de hipercelularidade, a alta contagem mitótica e o alto índice proliferativo com Ki-67 nos guiaram para o diagnóstico de lesão de alto grau. Apesar da marcação para S-100, aspecto histológico, negatividade para HMB-45 e melan-A, bem como a falta de identificação de outros sítios neoplásicos, afastamos a possibilidade de melanoma metastático.

O manejo terapêutico dessas lesões é incerto. A maioria dos estudos aponta a ressecção cirúrgica radical como tratamento de escolha. Não há consenso sobre a utilidade de radioterapia e quimioterapia adjuvantes. O estudo de Oliveira et al. não encontrou impacto na sobrevida de pacientes que fizeram uso de quimioterapia pós-operatória $(p=0,90)^{(7)}$. Mubarak et al.(6) sugerem a ressecção cirúrgica agressiva, seguida de radioterapia, como melhor tratamento e melhor impacto na sobrevida. O autor preconiza ainda ressecção de lesões recorrentes, sempre que factível| $(3,6,7)$.

O prognóstico dessas neoplasias é reservado e está relacionado com o grau histológico. Um estudo de 18 casos da Mayo Clinic encontrou queda na sobrevida de pacientes com tumores de alto grau comparados com os de baixo grau. Além disso, a comparação das curvas de sobrevida de paciente com glioblastomas e pacientes com sarcomas sugere evolução menos agressiva deste em relação ao primeiro ${ }^{(1,3,7)}$.

\section{Referências}

1. BISHOP, N. et al. A case of sarcoma of the central nervous system presenting as Guillain Barré syndrome. J Neurol Neurosurg Psychiatry, v. 46, n. 4, p. 352-4, 1983.

2. CAI, N. et al. A report of primary brain fibrosarcoma with literature review. J Neuro Oncology, v. 68, n. 2, p. 161-7, 2004.

3. KIM, S. et al. Primary intramedullary spinal sarcoma: a case report and review of the current literatures. J Korean Neurosurg Soc, v. 48, n. 2, p. 448-51, 2010.

4. MCDONALD, P. et al. Primary intracranial fibrosarcoma with intratumoral hemorrhage: neuropathological diagnosis with review of the literature. J Neuro Oncology, v. 35, n. 2, p. 133-9, 1997.
5. MENA, H. et al. Primary brain sarcomas: light and electron microscopic features. Cancer, v. 42, n. 3, p. 12982007, 1978.

6. MUBARAK, A. et al. Primary central nervous system sarcomas in children: clinical, radiological, and pathological features. Childs Nerv Syst, v. 19, n. 12, p. 808-17, 2003.

7. OLIVEIRA, A. M. et al. Primary sarcomass of the brain and sinal cord: a study of 18 cases. Am J Surg Pathol, v. 26, n. 8, p. 1056-63, 2002.

8. VATSAL, D. K. et al. Primary fibrossarcoma of brain. Neurol India, v. 48, n. 4, p. 396-8, 2000. 